\title{
ACS/WFC Pixel History, Bringing the Pixels Back to Science
}

\author{
Space Telescope Science Institute \\ 3700 San Martin Dr. \\ Baltimore, MD 21218 \\ email: dborncamp@stsci.edu
}

David Borncamp, Norman Grogin, Matthew Bourque and Sara Ogaz

\begin{abstract}
Excess thermal energy within a Charged Coupled Device (CCD) results in excess electrical current that is trapped within the lattice structure of the electronics. This excess signal from the CCD itself can be present through multiple exposures, which will have an adverse effect on its science performance unless it is corrected for. The traditional way to correct for this extra charge is to take occasional long-exposure images with the camera shutter closed. These images, generally referred to as "dark" images, allow for the measurement of thermal-electron contamination at each pixel of the CCD. This so-called "dark current" can then be subtracted from the science images by re-scaling to the science exposure times. Pixels that have signal above a certain value are traditionally marked as "hot" and flagged in the data quality array. Many users will discard these pixels as being bad. However, these pixels may not be bad in the sense that they cannot be reliably dark-subtracted; if these pixels are shown to be stable over a given anneal period, the charge can be properly subtracted and the extra Poisson noise from this dark current can be taken into account and put into the error arrays.
\end{abstract}

Keywords. Instrumentation, Dark Current, Hubble Space Telescope, HST, Hubble, Advanced Camera for Surveys, ACS, ACS/WFC

\section{Introduction}

Here we present an update to a pixel history study that analyzes every individual pixel of the 16 megapixel Advanced Camera for Surveys (ACS) Wide Field Channel (WFC) CCDs on board Hubble Space Telescope. Our time-series analysis allow pixels that were previously marked as bad to be brought back into the science image as a reliable pixel. We also present a statistic that classifies individual pixels into two categories: (1) "Stable" pixels that do not experience any odd dark behavior throughout a given anneal period and (2) "Unstable" pixels whose dark current values can vary significantly within a given anneal period. Very early analysis of these persistently hot pixels projected that they could make up as much as $6 \%$ of the detector by 2010 (Riess 2002). The implications of producing data containing this large amount of hot pixels make it necessary to determine if these pixels are actually reliable enough for science or not. Since the introduction of post flash into the darks gives us more signal to noise, we are also able to classify cold pixels as stable or unstable (Ogaz et al. 2015).

\section{Data}

For this analysis, we used every full frame calibration dark image taken for ACS/WFC that was used for creation of super darks for ACS/WFC. This resulted in 8,525 images used in the analysis taken from 146 anneal periods. We restructured the images into 
what we call "master column images," these images contain the same columns of each dark repeated in a time ordered fashion. This effectively puts the same pixel ordered in time along the $\mathrm{x}$-axis of the new image. This makes time series analysis much more computationally manageable since it reduces the amount of data needed to be read into and carried in memory by a factor of about 8,192 for ACS/WFC images.

To do this we first screened all dark images for cosmic rays by using ACSREJ (Lucas et al. 2016) with an association to contain all darks over an entire anneal period for maximum accuracy. The cosmic rays are then grown by 3 pixels in all directions to ensure that the effects from Charge Transfer Efficiency (CTE) inefficiencies (Anderson \& Bedin 2010) from the flux of the cosmic ray are not included in our analysis. Then all data was sorted by anneals and put into Hierarchical Data Format 5 files ("HDF5"; The HDF Group 1997-2016) which allowed us to break up the data more intuitively by separating data by amplifier and then columns as well as keeping track of all metadata associated with each dark for further analysis. The HDF5 framework also has other computational benefits for this type of analysis. Since we know exactly what size the data will be when we write it, we are able to define its size when we write it out and we are able to pack the file on disk exactly the way we need it. This allows for slightly more efficient in storage space than other data formats, i.e. FITS. HDF5 also allows us to store the data in chunks where the time axis is continuous, allowing for less striding when reading in the data and more efficient memory usage during analysis.

\section{Definition of Stability}

Since the dark current of a pixel can change with the temperature of the detector we define a pixel's stability over a given anneal period (which is roughly 4 weeks). We do this by utilizing the error arrays that accompany all WFC images and comparing their mean to the variance of the science arrays. The error extensions contain the poissonian errors from the collected signal, bias, and read noise to account for all errors in the individual pixels (Lucas et al. 2016) within a dark. The variance of this error is found and the mean of the error squared is subtracted to get the deviation from the mean. It is then normalized by the mean of the science array over the same time as illustrated in equation 3.1. This is applied over an entire anneal period and anything that has a value of greater than 2 is considered bad for this analysis. In this way we can determine the difference between the real variance and the expected variance and flag pixels accordingly.

$$
F=\frac{\text { Variance }(\text { ScienceArray })-\text { Mean }\left((\text { ErrorArray })^{2}\right)}{\text { Mean }(\text { Science })}+1
$$

\section{Classifying Pixels}

This definition of variance of a pixel leads to a natural classification of four different types of pixels:

- Hot Unstable: Average flux greater than the hot pixel threshold and varying by more than the allowed value.

- Cold Unstable: Low average flux and varying by more than the allowed value.

- Hot Stable: High average flux and stable throughout an anneal.

- Cold Stable: A good pixel with low average flux that is not significantly varying within in an anneal period. 


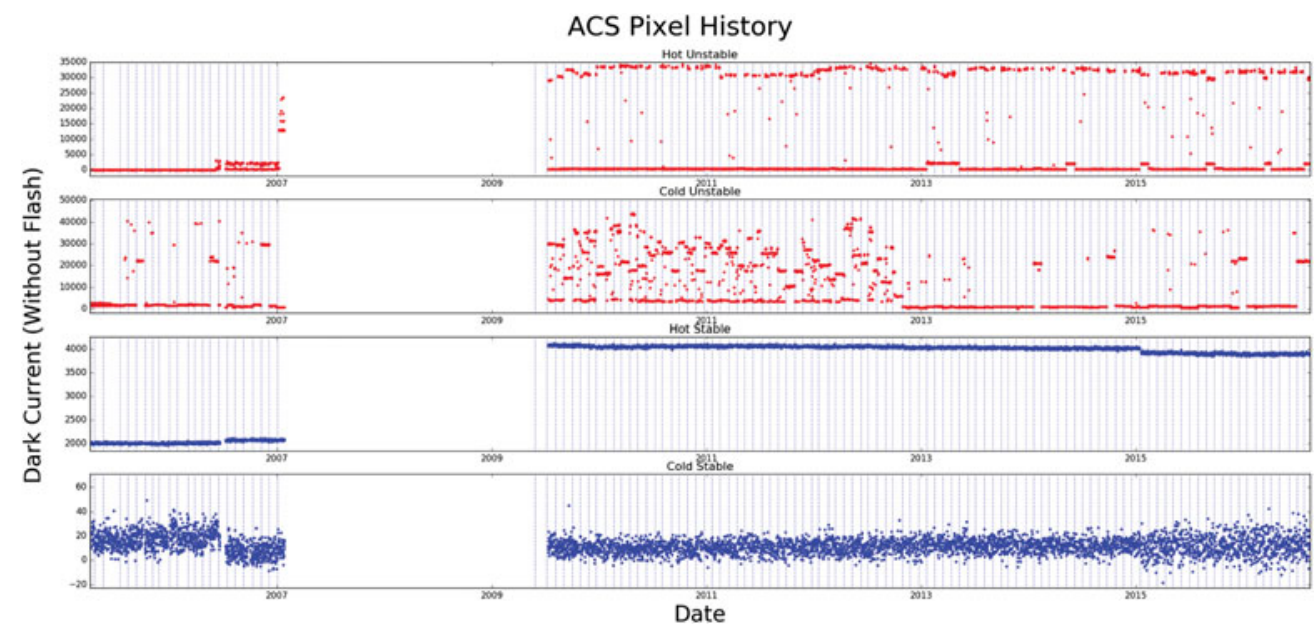

Figure 1. The different classifications of varying pixels from top to bottom. (1) A pixel whose high average dark current makes it hot in the traditional sense but it varies from dark exposure to exposure making it unstable. (2) A pixel whose average low value would allow it to be traditionally marked as cold but it is varying significantly so it should not be trusted. (3) A pixel that would be marked as Hot, but it is stable so it should be trusted. (4) A normal, cold stable pixel. Dotted vertical lines indicate anneal boundaries. The data gap starting in 2007 and ending in 2009 is when ACS was inoperable then repaired during Servicing Mission 4.

Table 1. Statistics for 0171825lj_drk.fits (useafter = Nov 042015 ) compared to figure of merit. Unstable is the total number of pixels marked as hot that are unstable.

Hot Unstable Hot Stable Hot Unstable Cold Unstable

\begin{tabular}{lccccc}
\hline \# Pixels & 506806 & 192103 & 500976 & 5830 & 186273 \\
\hline \% of detector & $3.02 \%$ & $1.15 \%$ & $2.98 \%$ & $0.04 \%$ & $1.10 \%$ \\
\hline \hline
\end{tabular}

Figure 1 shows the different types of pixels over the lifetime of the instrument with the vertical dashed lines being the anneal boundaries. It shows that some pixels dark flux can vary wildly within an anneal making reliable dark subtraction impossible. However, there are pixels that are traditionally marked as hot whose variance is low enough that it can be reliably dark subtracted. This figure also shows that different pixels can go between these classifications for different anneal periods. Some pixels are 'cured' after warming up the detector and may become unstable again in the future.

Table 1 shows the number of pixels that can be saved with this analysis by not being marked as bad. The hot row is how many pixels are currently being marked as hot by using a simple threshold. Most of these pixels, which are generally discarded by users, can be saved as seen in the Hot Stable column which shows how many pixels that are marked as hot but are stable as defined above. The unstable column shows how many pixels are defined as either hot unstable or cold unstable. These unstable pixels should not be trusted regardless of their being hot or cold, as the amount of dark current contributed to this pixel is unknown and cannot be corrected for.

It is worth noting that due to signal to noise issues, we can only classify cold unstable pixels after the ACS team started post flashing their darks in January 2015 (Ogaz et al. 2015) and this extra post flash shows increased noise in Figure 1. 


\section{Implications}

The incidence of hot pixels is expected to increase linearly with time as ACS/WFC continues to function and the rate of hot pixels has exceeded the amount of incident cosmic rays in a 1000 second exposure (Sirianni et al. 2006). Therefore it becomes important to be able to mitigate hot pixel effects. Since the majority of the persistently hot pixels are stable relative to their expected errors, and all poissonian errors are correctly propagated into the error arrays of each image, users may not want to discard the stably hot pixels depending on the acceptable errors for their science cases. Instead users should dark subtract these pixels and pay attention to the extra error that is contributed to the pixel value which is recorded in each error array of all ACS/WFC images. Using the information from the error array, users can make a more informed decision if they want to discard the hot pixels from their science rather than blindly discarding all hot pixels, as they can be trusted for some applications depending on the intended science.

As of this writing, the ACS/WFC Data Quality arrays are not flagged in this way, however the ACS team expects to incorporate flagging of these pixels described here in the near future. This analysis is also expected to continue as ACS/WFC acquires more data so we can accurately track variations in the dark current of individual pixels. It is also expected to be extended to other cameras on board HST to produce the best science possible.

\section{References}

Anderson, J. \& Bedin, L. 2010, PASP, 122, 1035

"HDF5"; The HDF Group. 1997-2016, Hierarchical Data Format, version 5

Lucas, R., et al. 2016, Advanced Camera for Surveys Data Handbook v. 8.0 (STScI)

Ogaz, S., Anderson, J., \& Golimowski, D. 2015, Post-Flash Calibration Darks for the Advanced Camera for Surveys Wide Field Channel (ACS/WFC), Tech. rep., Space Telescope Science Institute

Riess, A. 2002, The Projected Growth of Hot Pixels on ACS WFC, Tech. rep., Space Telescope Science Institute

Sirianni, M., Mutchler, M., \& Lucas, R. A. 2006, in The 2005 HST Calibration Workshop: Hubble After the Transition to Two-Gyro Mode, ed. A. M. Koekemoer, P. Goudfrooij, \& L. L. Dressel, 45 